\title{
Prognostic value of circulating tumor DNA in breast cancers
}

\author{
Yu Gui ${ }^{1}$, Xianchun Chen ${ }^{1}$, Shuman $\mathrm{Xu}^{1}$, Xiangdong Luo ${ }^{2,3}$ and Li Chen ${ }^{1,3}$ \\ ${ }^{1}$ Breast Disease Center, Southwest Hospital, Third Military Medical University, Chongqing, China \\ ${ }^{2}$ Burn Research Institute, Southwest Hospital, Third Military Medical University, Chongqing, China \\ ${ }^{3}$ National Key Laboratory of Trauma and Burns, Chongqing Key Laboratory of Disease Proteomics, Chongqing, China \\ Correspondence to: Li Chen, email: lichen2007@126.com
}

Keywords: circulating tumor DNA; meta-analysis; breast cancer

Received: August 23, $2017 \quad$ Accepted: January 24, $2018 \quad$ Published: February 13, 2018

Copyright: Gui et al. This is an open-access article distributed under the terms of the Creative Commons Attribution License 3.0 (CC BY 3.0), which permits unrestricted use, distribution, and reproduction in any medium, provided the original author and source are credited.

\section{ABSTRACT}

Circulating tumor DNA (ctDNA) comprises single- or double-stranded DNA that likely originates from cancer cells. The prognostic value of ctDNA detection in breast cancer patients is currently under debate. Here, we conducted the first comprehensive meta-analysis of the published literature on the prognostic relevance of ctDNA, in patients with early- and advanced-stage disease. The PubMed and Embase databases were searched for studies on ctDNA in breast cancer patients.The main outcomes analyzed were overall survival (OS) and disease-free survival (DFS) in early-stage breast cancer patients as well as progression-free survival (PFS) and OS in metastatic breast cancer patients. Pooled hazard ratios (HRs) and $95 \%$ confidence intervals (CIs) were calculated using random and fixed-effects models. A total of 16 studies involving 2070 participants were identified as eligible for inclusion in our meta-analysis, and the results of the pooled analysis showed that the presence of ctDNA was significantly associated with poor OS (HR $=1.98 ; 95 \% C I$ : $1.38-2.83, P=0.0002)$ and DFS/PFS ( $H R=1.87 ; 95 \% \mathrm{CI}: 1.35-2.60, P=0.0002)$ in the total breast cancer population. Furthermore, subgroup analysis revealed that associations between the presence of ctDNA and the outcome endpoints (OS and PFS) were significant within the metastatic breast cancer patient group and in patients with TP53 or ESR1 mutations in ctDNA.The TP53 and ESR1 mutations in ctDNA are potential prognostic biomarkers and promising therapeutic targets for advanced breast cancer.

\section{INTRODUCTION}

Breast cancer is the most frequently diagnosed cancer worldwide and was the leading cause of cancer death in women in 2012 [1]. Increased real-time monitoring of cancer evolution and therapeutic efficacy might reduce the breast cancer mortality rate [2]. Currently, breast cancer progression in patients is mainly monitored using serial physical examinations and radiographic imaging [3]. These strategies are largely effective for the detection of breast cancer progression when there are relatively significant changes. However, these two modalities often fail to rapidly detect changes in tumor burden when the patients have asymptomatic diseases, such as ductal carcinoma in situ $[4,5]$. Cancer is associated with mutated genes, and analysis of tumor-associated genetic alterations is increasingly used for diagnostic, prognostic and treatment purposes. The genetic profile of solid tumors is currently obtained from surgical or biopsy specimens; however, the latter procedure cannot be routinely performed due to its invasive nature. A liquid biopsy, such as a blood sample, can provide the genetic landscape of all cancerous lesions (primary and metastases) and allow systematic tracking of genomic evolution [6]. Therefore, non-invasive biomarkers that can guide personalized treatment, predict prognosis and facilitate reliable follow-up are needed. Potential noninvasive biomarkers, such as cancer antigen 15-3 and circulating tumor cells, have been studied in breast cancer patients. However, these markers have limited utility in clinical settings due to their low sensitivity and small range of dynamic change compared to the magnitude of change in tumor burden [7-9]. 
Circulating tumor DNA (ctDNA) comprises singleor double-stranded DNA that likely originates from cancer cells through the process of necrosis and apoptosis, and ctDNA exists in the plasma or serum [10-12]. ctDNA contains specific tumor mutations (i.e., TP53, PIK3CA and $E S R$ l) in the cell-free DNA fraction of a given patient [13]. The presence of ctDNA in the blood of patients with breast cancer have been used to monitor the treatment response, detect resistance and relapse, and provide prognostic information [14]. Following whole-genome sequencing, ctDNA can be detected in more than $75 \%$ of patients with advanced disease and $50 \%$ of patients with early-stage disease [7]. Furthermore, the assay has a high sensitivity and specificity of $90 \%$ and $100 \%$, respectively, showing the feasibility of detecting DNA mutations in the plasma of early-stage breast cancer patients, including patients with minimal, clinically undetectable disease [15]. Moreover, ctDNA can be used as a tool to monitor changes in tumor burden among metastatic breast cancer patients undergoing systemic therapy [16]. Thus, ctDNA is currently being considered as a non-invasive source for the identification of targetable genomic alterations that can serve as prognostic predictors and improve the survival of breast cancer patients. Recently, multiple studies have investigated the correlation between the presence of ctDNA and prognosis among breast cancer patients. An overwhelming majority of the evidence has shown a correlation between poor prognosis and the presence of ctDNA in breast cancer [17-20], but several studies have failed to draw similar conclusions [21-22]. Therefore, the prognostic value of ctDNA in breast cancer patients remains controversial.

Because the prognostic value of ctDNA in breast cancer patients is expected to be further characterized in future studies, we conducted the first comprehensive meta-analysis of the published literature on this topic. We evaluated the prognostic value of ctDNA status (presence vs. absence) on disease-free survival (DFS) among early breast cancer patients, progression-free survival (PFS) among metastatic breast cancer patients and overall survival (OS) in early and metastatic breast cancer patients. Specifically, we performed subgroup analyses to evaluate whether tumor stage, gene mutations and the time point of blood collection influence the prognostic value of ctDNA.

\section{RESULTS}

\section{Included studies}

Initially, 745 studies were identified from the database search, and after evaluating the titles and abstracts, 665 studies were excluded based on the selection criteria. Subsequently, 80 potentially relevant full-text articles were reviewed further. Among them, 64 studies were excluded: twenty-eight studies with no or insufficient data; four studies that used the same or overlapping data; ten articles comprising reviews, commentaries or letters; four studies that included gene analysis; twelve articles that evaluated methylation; three studies that investigated DNA integrity; two studies that included a HER2 amplification analysis; and one case report. Ultimately, 16 studies were identified as eligible for inclusion in our systematic review. Figure 1 summarizes the overall literature search and selection criteria.

\section{Study characteristics}

The general characteristics of the participants from all included studies are presented in Table 1. A total of 2070 patients (ranging from 16 to 541 per study) were included in these studies. One thousand seventy-five early-stage (M0) and 995 metastatic (M1) breast cancer patients were enrolled in 10 and 7 studies, respectively. Of the fourteen studies that reported gene mutations, 5, 5 and 7 analyzed ESR1, PIK3CA and TP53 mutations, respectively. Nine and six studies collected blood samples before and after any systemic adjuvant therapy, respectively. The quality scores of all studies were at least 7 (Table 1).

\section{Global analysis of the association between ctDNA and survival}

Eleven studies were included in the analysis of the effect of ctDNA on DFS/PFS of breast cancer patients. The results of our meta-analysis revealed that ctDNA-positive patients were significantly associated with worse DFS/ PFS compared to ctDNA-negative patients. The pooled HR value was 1.87 (95\% CI: $1.35-2.60, P=0.0002)$ (Figure 2A). Due to significant heterogeneity among the studies $\left(P<0.0001, I^{2}=68 \%\right)$, the random-effects model was applied. Data on OS were extracted from nine studies. A random-effects model $\left(I^{2}=69 \%, P=0.0004\right)$ was used to calculate the HR of OS in breast cancer patients. The meta-analysis results showed that ctDNA-positive patients were significantly associated with worse OS compared to ctDNA-negative patients, with a pooled HR value of 1.98 (95\% CI: 1.38-2.83, $P=0.0002$ ) (Figure 2B).

\section{Subgroup analysis of the association between ctDNA and breast cancer survival based on tumor stage}

Subgroup analyses stratified by tumor stages showed that ctDNA-positive metastatic breast cancer patients were significantly associated with worse PFS and OS compared with control patients, with pooled HR values of $1.66(95 \%$ CI: $1.21-2.29, P=0.002)$ and 2.20 (95\% CI: $1.34-3.60$, $P=0.002$ ), respectively (Figure 3 ). Heterogeneity among the studies was moderate $\left(I^{2}=60 \%\right.$ for PFS; $I^{2}=55 \%$ for OS). However, the estimated pooled HR showed that the presence of ctDNA was not associated with DFS and OS in the early-stage breast cancer patients (HR, 1.97; 95\% 
CI: $0.89-4.34 ; P=0.09$ and HR, $1.87 ; 95 \%$ CI: 0.94-3.74; $P=0.07$, respectively) (Figure 3 ). Because heterogeneity among the studies was significant $\left(P=0.0004, I^{2}=78 \%\right.$ for DFS and $P=0.0006, I^{2}=77 \%$ for OS), the randomeffects model was applied.

\section{Subgroup analysis of the effect of gene mutations in ctDNA on breast cancer survival}

While evaluating the relevance of gene mutations, our results showed that patients with ESR1 and TP53 mutations in ctDNA were significantly associated with worse DFS/ PFS compared with control patients, with a pooled HR of 1.79 (95\% CI: 1.16-2.78; $P=0.009)$ and 2.20 (95\% CI: 1.67-2.90; $P<0.00001$ ), respectively (Figure 4A). Our meta-analysis did not show an association between PIK3CA mutations and DFS, as shown in Figure 4A. However, the patients with ESR1 and TP53 mutations in ctDNA were significantly associated with only worse OS compared to control patients, with a pooled HR of 1.59 (95\% CI: 1.28-1.98; $P<0.0001)$ and 2.82 (95\% CI: $1.82-4.36 ; P<$ $0.00001)$, respectively; patients with $P I K 3 C A$ mutations in ctDNA did not demonstrate an association with OS (Figure 4B). When we analyzed the different TP53 mutation sites, we found that patients with TP53 mutations in exons 5-8 were significantly associated with worse DFS/PFS and OS compared to control patients, with a pooled HR of 2.32 (95\% CI: $1.55-3.46 ; P<0.0001)$ and 2.52 (95\% CI: 1.49 4.26; $P=0.0006)$, respectively. However, patients with TP53 mutations in all exons were significantly associated with worse OS compared to control patients, with a pooled HR of 3.60 (95\% CI: 1.65-7.86; $P=0.001$ ) (Figure 5). When we analyzed the different ESR 1 mutation sites, we found that the patients with mutations in amino acids 537 and 538 were significantly associated with worse DFS/ PFS and OS compared to control patients, with a pooled HR of 1.91 (95\% CI: $1.05-3.49 ; P=0.04)$ and $1.59(95 \%$ CI: $1.28-1.98 P<0.0001)$, respectively; ESR1 mutations at any site were not significantly associated with DFS (Supplementary Figure 1).

\section{Subgroup analysis of the effect of the sampling time point, molecular classification and detection method on the prognostic value of ctDNA for breast cancer survival}

As shown in Table 2, subgroup analyses stratified by the blood-sampling time point, molecular classification and detection method confirmed that ctDNA presence was a strong prognostic indicator in all sampling-time

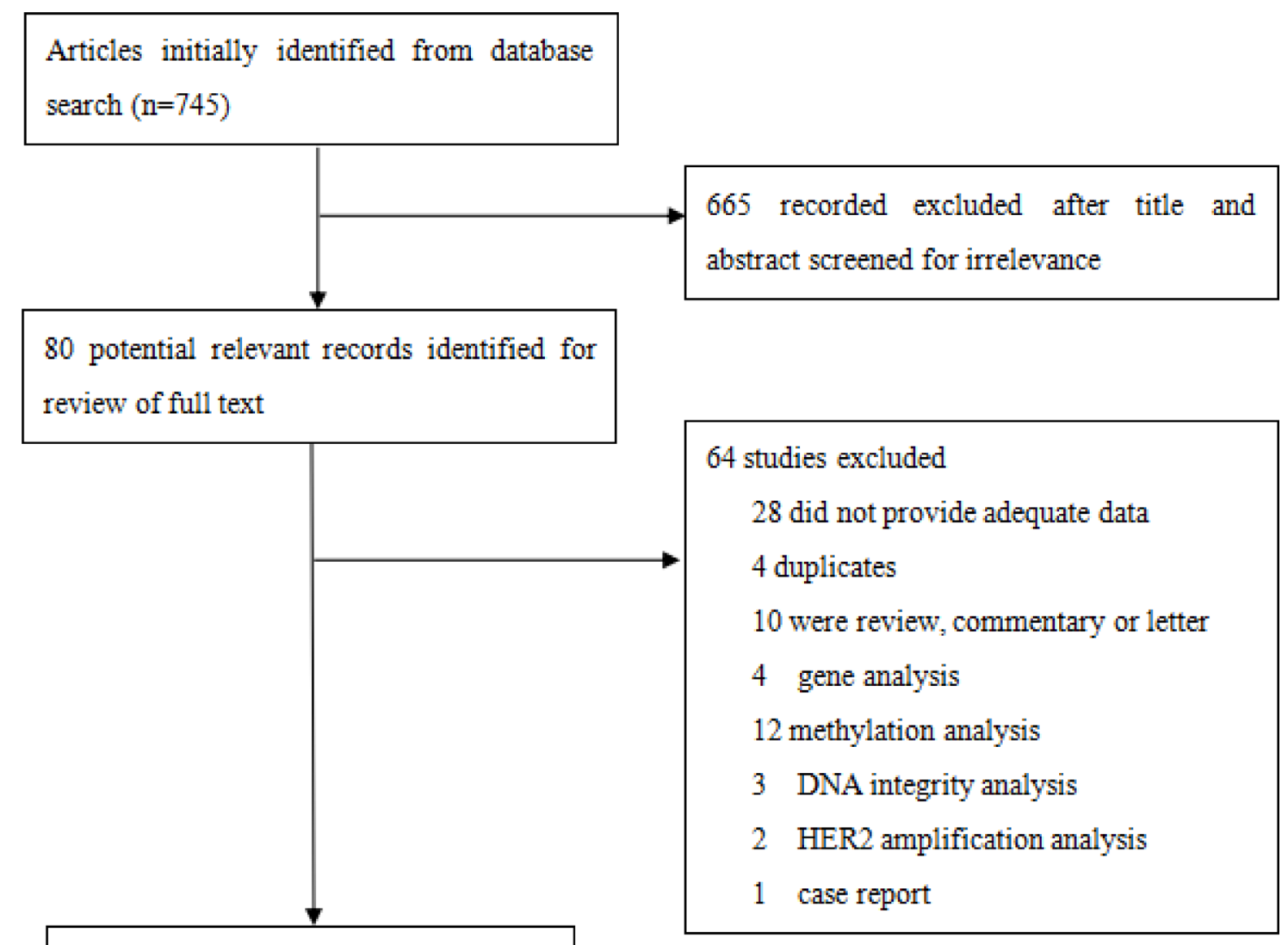

16 records included in meta-analysis

Figure 1: Flow diagram depicting the literature search and criteria for the selection of the included studies. 
Table 1: Characteristics of studies included in the meta-analysis

\begin{tabular}{|c|c|c|c|c|c|c|c|c|c|c|}
\hline Ref. & First author & Year & $\begin{array}{l}\text { Country/ } \\
\text { region }\end{array}$ & $\begin{array}{l}\text { NO. Of } \\
\text { patients }\end{array}$ & Stage & $\begin{array}{c}\text { Detection } \\
\text { method }\end{array}$ & $\begin{array}{c}\text { Mutation } \\
\text { Gene }\end{array}$ & $\begin{array}{l}\text { Sampling } \\
\text { time }\end{array}$ & NOS & Outcome \\
\hline [17] & Garcia & 2006 & Spain & 142 & $\mathrm{M}_{0}$ & PCR & TP53 Mutations & Baseline & 9 & DFS, OS \\
\hline$[18]$ & Liang & 2015 & USA & 100 & $\mathrm{M}_{0}$ & NA & $\begin{array}{c}\text { TP53 Mutation } \\
\text { PIK3CA Mutation }\end{array}$ & NA & 8 & PFS \\
\hline [19] & Oshiro & 2015 & Japan & 313 & $\mathrm{M}_{0}$ & $\mathrm{dPCR}$ & PIK3CA Mutation & Baseline & 7 & RFS, OS \\
\hline [20] & Schiavon & 2015 & UK & 171 & $\mathrm{M}_{1}$ & $\mathrm{dPCR}$ & ESR1 Mutation & Treatment & 7 & PFS \\
\hline [21] & Madic & 2014 & France & 40 & $\mathrm{M}_{1}$ & PCR & TP53 Mutation & Treatment & 8 & PFS, OS \\
\hline [22] & Gyanchandani & 2016 & USA & 16 & $\mathrm{M}_{1}$ & $\mathrm{dPCR}$ & ESR1 Mutation & Treatment & 9 & PFS, OS \\
\hline [23] & Chandarlapaty & 2016 & USA & 541 & $\mathrm{M}_{1}$ & dPCR & ESR1 Mutation & Baseline & 7 & PFS, OS \\
\hline [24] & Garcia-Murillas & 2015 & UK & 55 & $\mathrm{M}_{0}$ & $\mathrm{dPCR}$ & PIK3CA Mutation & $\begin{array}{l}\text { Baseline } \\
\text { Treatment }\end{array}$ & 8 & DFS \\
\hline$[25]$ & Nakauchi & 2016 & Japan & 17 & $\mathrm{M}_{1}$ & NGS & $\begin{array}{l}\text { TP53 Mutation } \\
\text { PIK3CA Mutation }\end{array}$ & Treatment & 7 & OS \\
\hline [26] & Olsson & 2015 & Sweden & 20 & $\mathrm{M}_{0}$ & $\mathrm{dPCR}$ & NA & Baseline & 9 & OS \\
\hline [27] & Riva & 2017 & France & 46 & $\mathrm{M}_{0}$ & $\mathrm{dPCR}$ & TP53 Mutations & $\begin{array}{l}\text { Baseline } \\
\text { Treatment }\end{array}$ & 8 & DFS, OS \\
\hline [28] & Shao & 2001 & China & 126 & $\mathrm{M}_{0}$ & PCR & TP53 Mutations & Baseline & 7 & DFS, OS \\
\hline [29] & Silva & 2002 & Spain & 147 & $\mathrm{M}_{0}$ & PCR & TP53 Mutations & Baseline & 7 & DFS \\
\hline [30] & Spoerke & 2016 & USA & 168 & $\mathrm{M}_{1}$ & $\mathrm{dPCR}$ & $\begin{array}{l}\text { ESR1 Mutation } \\
\text { PIK3CA Mutation }\end{array}$ & Baseline & 7 & PFS \\
\hline [31] & Takeshita & 2015 & Japan & 49 & $\mathrm{M}_{0}$ & $\mathrm{dPCR}$ & PIK3CA Mutation & NA & 7 & RFS, OS \\
\hline [32] & Takeshita & 2016 & Japan & 119 & $\mathrm{M}_{0}, \mathrm{M}_{1}$ & $\mathrm{dPCR}$ & ESR1 Mutation & NA & 8 & DFS, OS \\
\hline
\end{tabular}

Note: NGS: Next generation sequencing; dPCR: digital PCR

groups, the estrogen receptor-positive $(\mathrm{ER}+)$ breast cancer subgroup and the subgroup designated "PCR". The prognostic values of ESR1 mutations in ctDNA for DFS and OS were significant in the ER+ breast cancer group, with a pooled HR of 1.57 (95\% CI: 1.02-2.39; $P=0.04)$ and 1.59 (95\% CI: $1.28-1.98 ; P<0.0001)$, respectively. However, $P I K 3 C A$ mutations in ctDNA were not significantly associated with DFS among ER+ breast cancer patients. Additionally, the prognostic value of ctDNA for DFS and OS was not significant in the triplenegative breast cancer subgroup.

We also analyzed the data to determine the association between the detection method and DFS or OS in ctDNA-positive patients, as shown in Table 2. We specifically compared the associations of detection by droplet digital PCR (ddPCR), PCR or next generation sequencing (NGS) with DFS or OS. We observed that ctDNA detection in patients by PCR was significantly associated with worse DFS and OS outcomes (HR $=2.12$, 95\% CI: $1.46-3.09 ; P<0.0001$ and $\mathrm{HR}=2.54,95 \%$ CI: $1.58-4.07 ; P=0.0001$, respectively) compared to detection by other methods. When ctDNA was detected by ddPCR, the prognostic value of ctDNA for DFS was significant $(\mathrm{HR}=1.79,95 \% \mathrm{CI}: 1.10-2.92 ; P=0.02)$; however, the prognostic value of ctDNA for OS was not significant. Only one study reported that ctDNA was detectable by NGS. Therefore, additional data on ctDNA detection by NGS must be analyzed.

\section{Heterogeneity analysis}

The heterogeneity tests indicated significant heterogeneity among the studies, with $I^{2}$ scores of $68 \%$ and $69 \%$ for DFS and OS, respectively (Figure 2); generally, $I^{2}>50 \%$ indicated heterogeneity. Therefore, we used the random-effects model to calculate combined effect indicators. Heterogeneity was significantly reduced when the studies included TP53 mutations in ctDNA with $I^{2}=0 \%$ (Figure 4), suggesting that the classification of the mutated gene might contribute to heterogeneity. A sensitivity analysis was conducted to investigate the influence of each study; no significant difference was observed after removing any single study, suggesting that the conclusions are stable. The risk of bias of individual studies is summarized in Figure 6. Four studies provided details of their random sequence generation methods, and two studies exhibited a high risk of bias due to the blinding of participants.

\section{DISCUSSION}

The notable need to screen the ctDNA fragments in blood samples to enable accurate prediction of prognosis among breast cancer patients prompted us to undertake this systematic review and meta-analysis. Using the strict inclusion and exclusion criteria for patient selection, we identified 16 high-quality studies, and ctDNA assessment 
was organized according to prognostic predictability regarding PFS/DFS and OS in breast cancer patients. Our meta-analysis demonstrated that ctDNA was associated with poor prognosis with statistically significant results for both PFS/DFS and OS in breast cancer patients. A further subgroup analysis showed that ctDNA (TP53, PIK3CA and ESR 1 mutations) was significantly associated with poorer PFS and OS outcomes in metastatic breast cancer patients than in early-stage breast cancer patients. Additionally, we observed that only the patients with TP53 or ESR1 mutations in ctDNA were significantly correlated with both poor PFS/DFS and OS.

In this meta-analysis, we found that ctDNA (TP53, PIK3CA and ESR 1 mutations) was significantly associated with worse DFS/PFS and OS in breast cancer patients. Researchers tested blood samples from various cancer patients and found that ctDNA was present at significantly different levels among patients with different stages of cancer. Specifically, patients with advanced-stage breast cancers had a higher level of ctDNA than patients with early-stage cancers [7], indicating that ctDNA could be significantly correlated with distant metastasis and prognosis. Additionally, ctDNA (PIK3CA mutation) could be used to monitor relapse status in relapsing and non-relapsing patients, resulting in a 10-month leadtime on the detection of relapse compared with the conventional follow-up strategy [24]. Therefore, ctDNA (TP53, PIK3CA and ESR1 mutations) can be an important

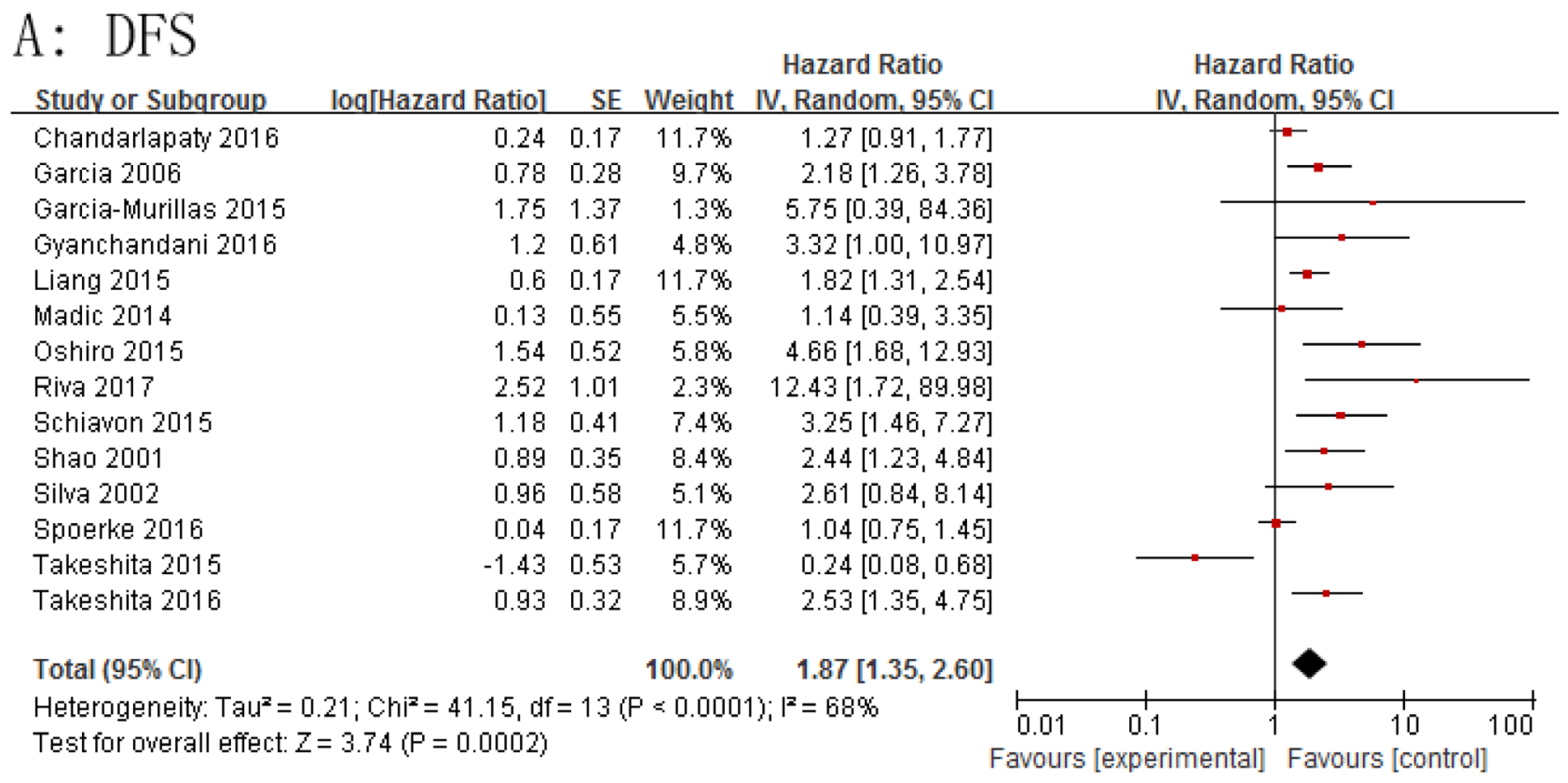

\section{B: OS}

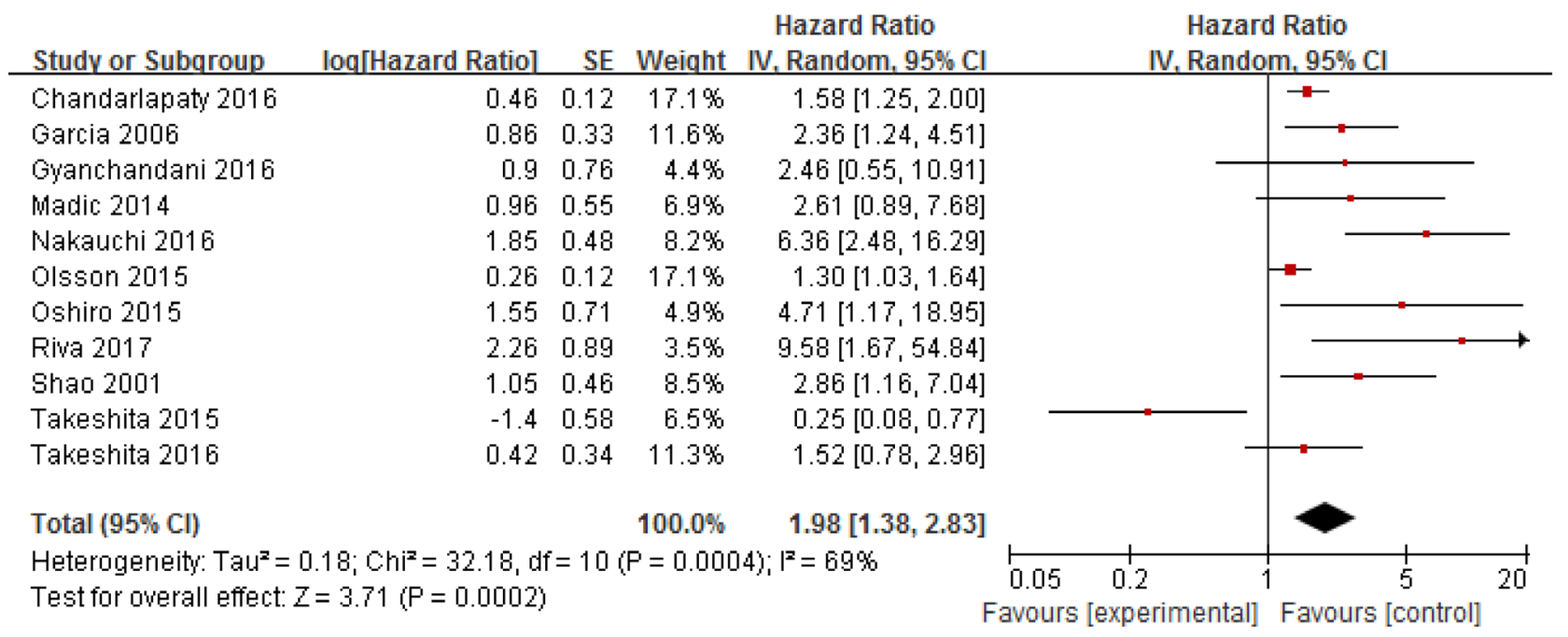

Figure 2: Meta-analysis of the HRs for DFS and OS among breast cancer patients depending on the absence or presence of ctDNA. (A) DFS; and (B) OS. 

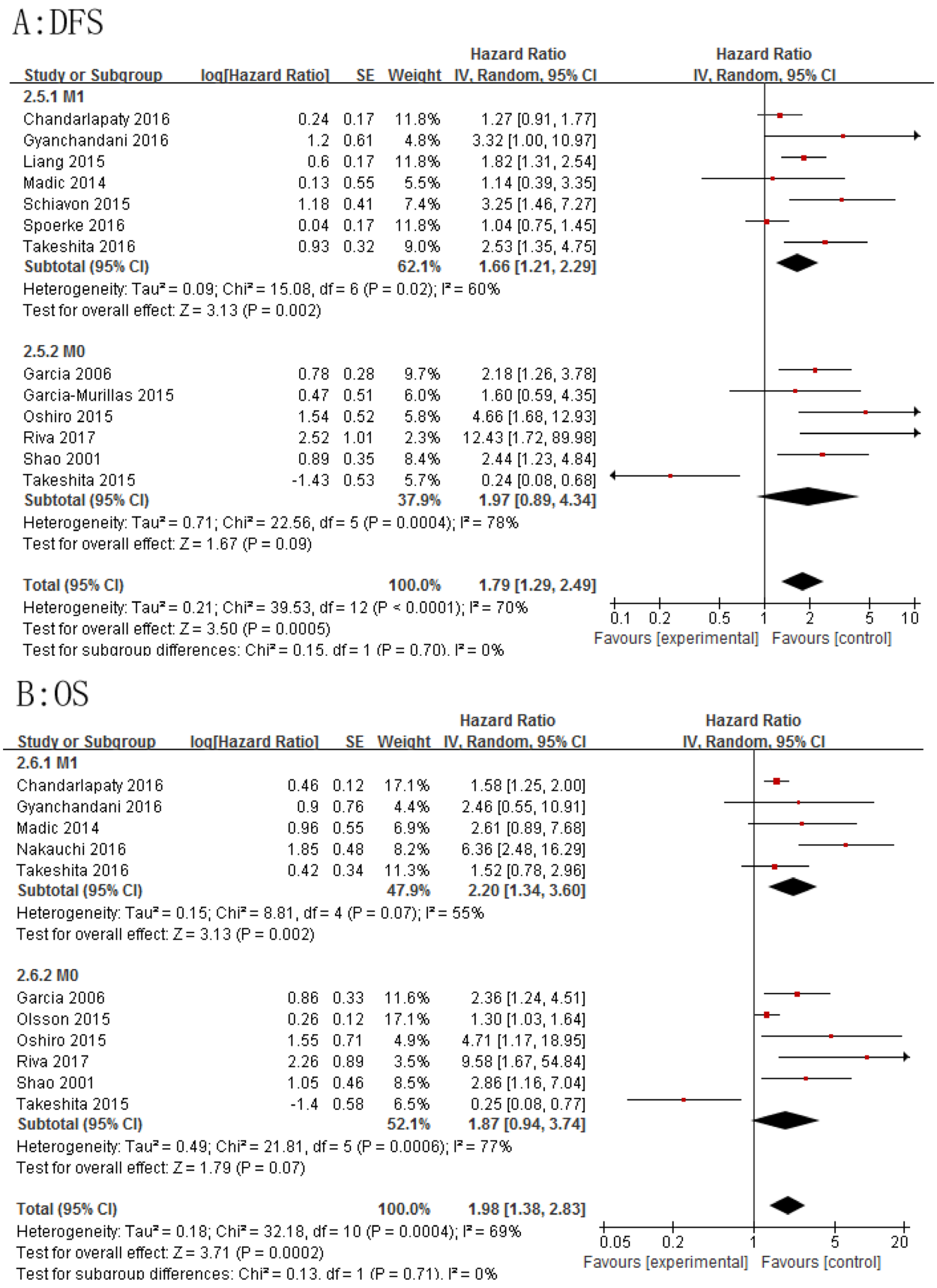

Figure 3: Meta-analysis of the HRs for DFS and OS among early-stage and metastatic breast cancer patients depending on the absence or presence of ctDNA. (A) DFS for early-stage disease (M0) and metastatic disease (M1). (B) OS for early-stage disease (M0) and metastatic disease (M1). 

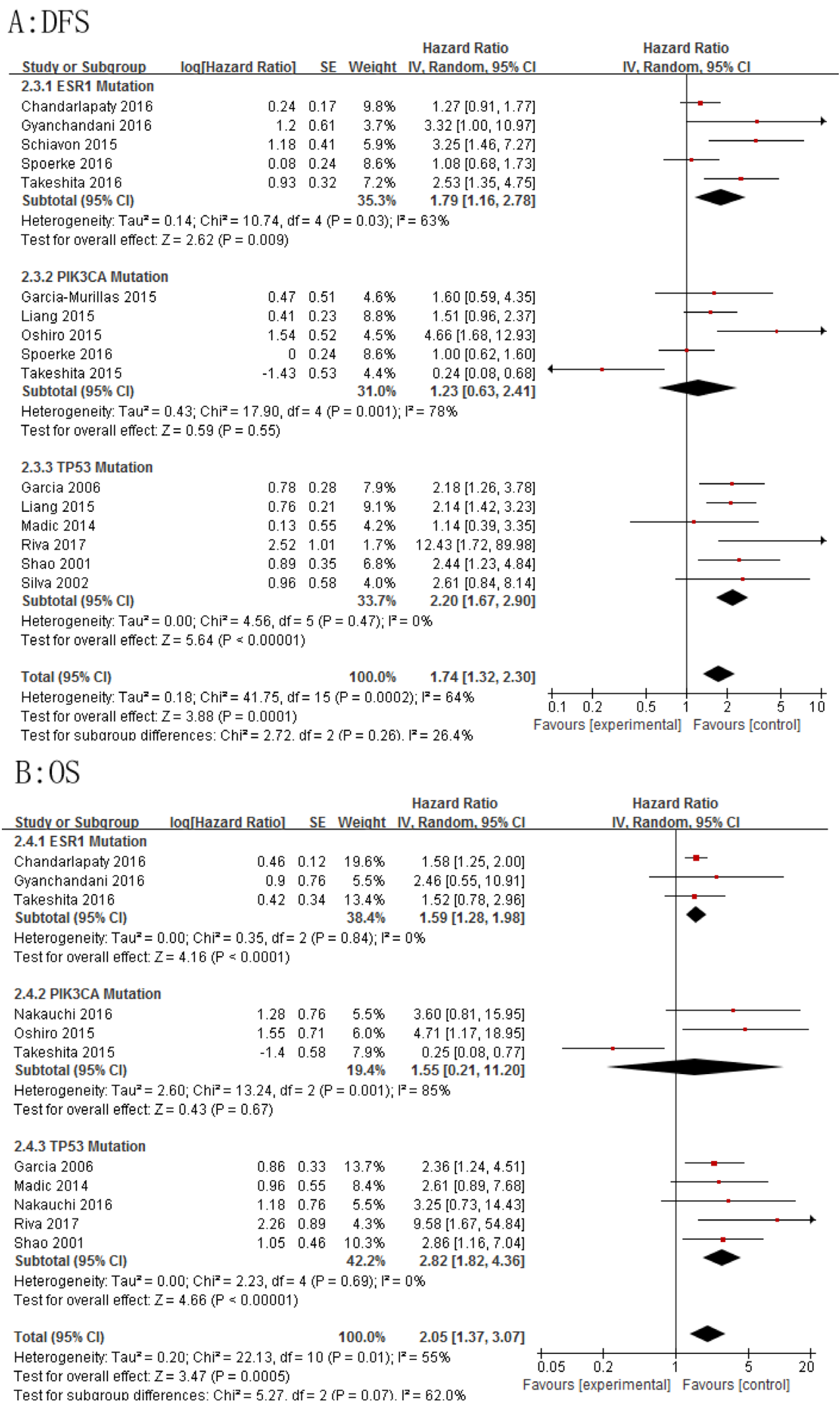

Figure 4: Meta-analysis of the HRs for DFS and OS regarding the influence of mutated genes on the prognostic impact of ctDNA. (A), DFS. (B) OS. 
indicator of worse outcome and can be used to monitor disease progression after primary treatment among breast cancer patients.

Regarding tumor stage, the presence of ctDNA (TP53, PIK3CA and ESR1 mutations) in breast cancer patients had a negative influence on clinical outcome in the metastatic cancer group, with consistent results for OS and PFS. Although the presence of ctDNA (PIK3CA mutation) was not significantly correlated with $\mathrm{OS}$ or
DFS in the early-stage breast cancer group, the presence of ctDNA ( $P I K 3 C A$ mutation) was shown to be a favorable survival biomarker and was significantly associated with DFS or OS in early-stage triple-negative breast cancer patients [31]. The pooled results showed significant associations between ctDNA (TP53, PIK3CA and ESR1 mutations) and poor OS or DFS in early-stage breast cancer patients; and when we excluded this study, the pooled HRs and 95\% CIs were 2.38 (95\% CI: 1.65-3.44;
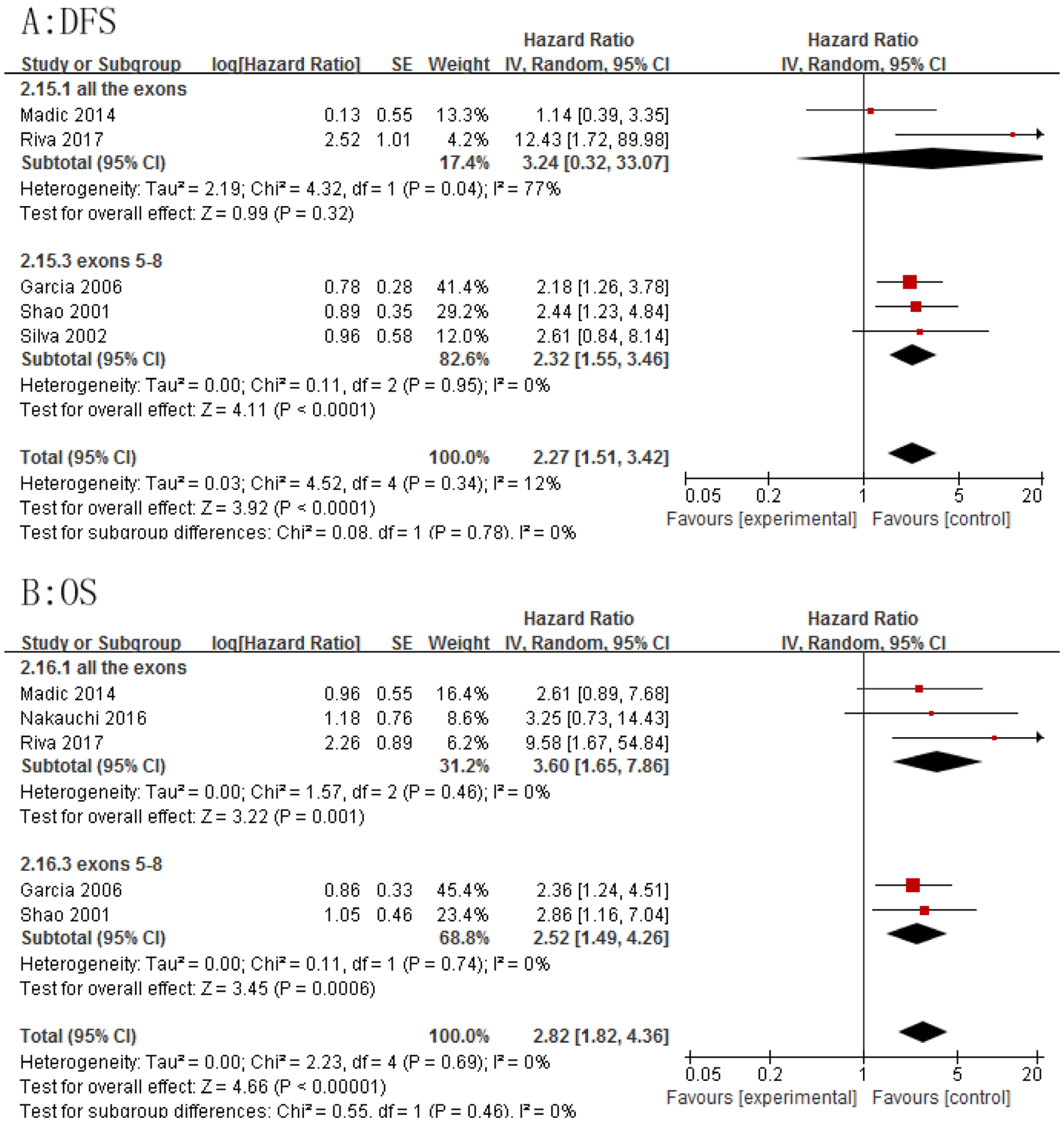

Figure 5: Meta-analysis of the HRs for DFS and OS regarding the influence of the TP53 mutation site on the prognostic impact of ctDNA. (A) DFS. (B) OS. 
Table 2: Results of subgroup analyses for the effects of ctDNA on disease-free and overall survival

\begin{tabular}{|c|c|c|c|c|c|c|}
\hline \multirow[b]{2}{*}{ Study selection } & \multicolumn{3}{|c|}{ DFS } & \multicolumn{3}{|c|}{ OS } \\
\hline & df & $\begin{array}{l}\text { HR }(95 \% \text { CI; } \\
\text { random effects) }\end{array}$ & $P$ & df & $\begin{array}{l}\text { HR }(95 \% \text { CI; } \\
\text { random effects) }\end{array}$ & $\boldsymbol{P}$ \\
\hline \multicolumn{7}{|l|}{ Sampling time } \\
\hline Baseline & 6 & $1.97[1.19,3.24]$ & 0.008 & 5 & $2.12[1.26,3.57]$ & 0.004 \\
\hline Treatment & 5 & $4.11[1.65,10.22]$ & 0.002 & 4 & $4.51[2.10,9.68]$ & 0.0001 \\
\hline \multicolumn{7}{|c|}{ Molecular classification } \\
\hline TNBC & 4 & $2.31[0.35,15.13]$ & 0.38 & 3 & $1.71[0.23,12.88]$ & 0.60 \\
\hline \multirow[t]{2}{*}{$\mathrm{ER}+$} & $4^{a}$ & $1.57[1.02,2.39]$ & 0.04 & $3^{\mathrm{b}}$ & $1.59[1.28,1.98]$ & $<0.0001$ \\
\hline & $2^{\mathrm{c}}$ & $3.53[0.21,58.53]$ & 0.38 & & & \\
\hline \multicolumn{7}{|l|}{ Detection method } \\
\hline dPCR & 9 & $1.79[1.10,2.92]$ & 0.02 & 7 & $1.51[1.01,2.26]$ & 0.05 \\
\hline PCR & 4 & $2.12[1.46,3.09]$ & $<0.0001$ & 3 & $2.54[1.58,4.07]$ & 0.0001 \\
\hline NGS & & & & 1 & $6.36[2.48,16.29]$ & 0.0001 \\
\hline
\end{tabular}

Note: $\mathrm{M}^{0}$ : early-stage patients; $\mathrm{M}^{1}$ : metastatic patients

${ }^{a}$ For the DFS of the ER positive breast cancer patients with ESR1 mutation in ctDNA

${ }^{b}$ For the OS of the ER positive breast cancer patients with ESR1 mutation in ctDNA

${ }^{\mathrm{C}}$ For the DFS of the ER positive breast cancer patients with PIK3CA mutation in ctDNA

$P<0.00001)$ and 2.08 (95\% CI: $1.20-3.61 ; P=0.009)$, respectively. We believe that these differences were due to the inclusion of patients who had triple-negative breast cancer in this study. Therefore, additional investigations are required to clarify the role of ctDNA (TP53, PIK3CA and ESR1 mutation) as a prognostic biomarker for earlystage breast cancer.

We also analyzed the relationships between different gene mutations in ctDNA and survival in breast cancer patients. Our meta-analysis confirmed that ctDNA with TP53 mutations in exons 5-8 and ESR1 mutations in amino acids 537 or 538 were associated with poor prognosis among breast cancer patients. Therefore, detection of ctDNA with TP53 mutations in exons 5-8 and ESR1 mutations in amino acids 537 or 538 in breast cancer patients can help monitor disease progression and be an important indicator of worse outcome.

Cancer genome characterization efforts have shown that only a few genes are frequently mutated in breast cancer (e.g., TP53, PIK3CA and ESR 1) and that a larger number of cancer genes are occasionally mutated [3337]. ctDNA has the potential to identify the emergence of mutations. Additionally, ctDNA was more sensitive and specific than other circulating blood biomarkers when we detected gene mutations (i.e., TP53, PIK3CA and ESR1) in ctDNA in primary breast cancer patients [13]. Previous studies have reported that TP53 mutations are found in more than $80 \%$ of ctDNA samples. Mutations in the ESRI gene are acquired in approximately $20 \%$ of breast cancers patients treated with endocrine agents and constitute a mechanism of resistance to aromatase inhibitors [38-40].
Regarding gene mutations in ctDNA, we analyzed the associations of three gene mutations in ctDNA and found that the presence of TP53 or ESR1 mutations in the ctDNA of breast cancer patients had a negative influence on clinical outcome and that PIK3CA mutations in the ctDNA of breast cancer patients had no influence on clinical outcome, with consistent results for DFS and OS. This finding indicates that the presence of TP53 or ESR1 mutations in ctDNA is a predictor of poor prognosis in breast cancer patients, but the presence of PIK3CA mutations in ctDNA is not a predictor of prognosis in breast cancer patients. Additionally, we found that ctDNA with TP53 mutations only in exons 5-8 and ESR1 mutations in amino acids 537 or 538 were unfavorable prognostic predictors for breast cancer patients. Mutations in $\mathrm{p} 53$ suppress the regulatory functions of the protein and are the most common genetic change in breast cancer, with a frequency of approximately $30 \%$ (range 15-71\%) [41]. p53 mutations are characterized by a high prevalence of missense mutations primarily in exons 5-8, which encode the DNA-binding domain. Previous studies have shown that TP53 mutations in exons 5-8 comprise $90 \%$ of the mutations reported in breast cancer [42, 43]. In the future, we will conduct additional clinical research on ctDNA, as we did not analyze the prognostic values of ctDNA with TP53 mutations in exons 5-8 and ESR1 mutations in amino acids 537 or 538 in patients with other cancers

Approximately $70 \%$ of breast tumors are ER+, which usually suggests sensitivity to hormonal blockage. The key role of ER gene (ESR1) mutations in aromatase inhibitor (AI) resistance was recently investigated. The 


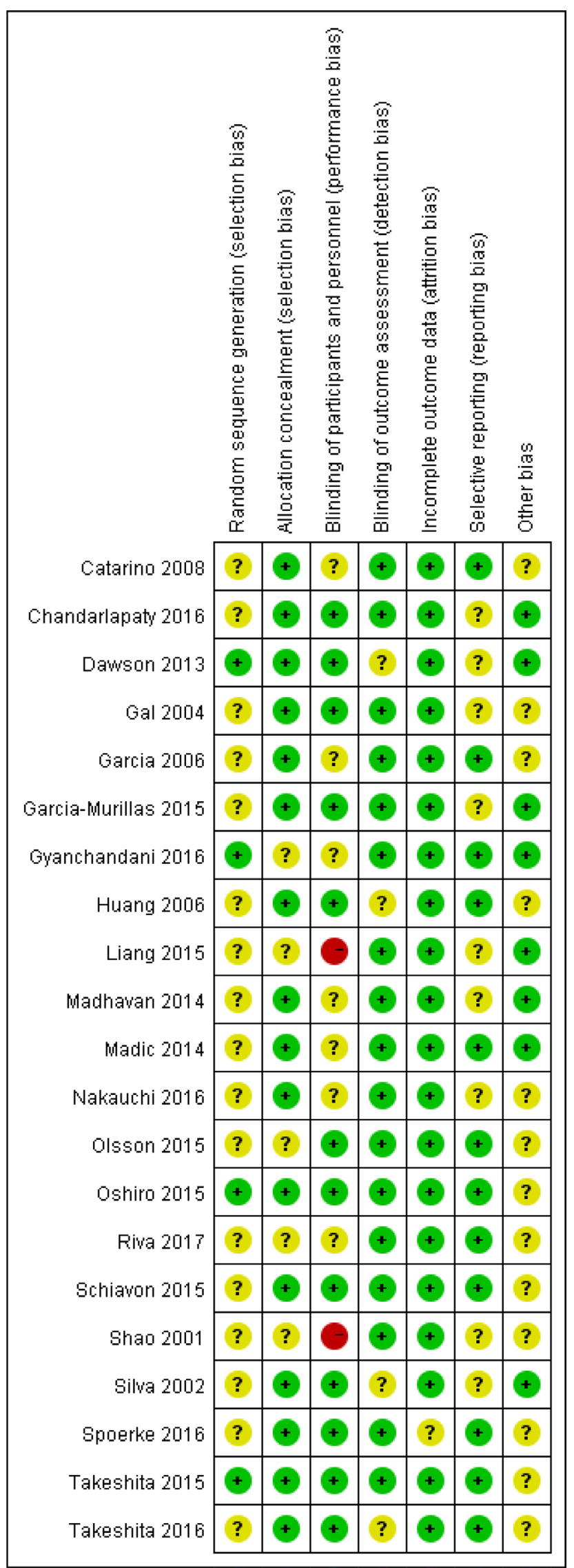

Figure 6: Risk of bias among the studies included in the meta-analysis. 
mutations in amino acids 537 and 538 account for over $80 \%$ of all ESR1 mutations; these mutations usually modify the ligand binding domain of the ER and lead to ligand-independent receptor activity [44]. Thus, ctDNA was a predictor of poor prognosis in ER+ breast cancer patients.

Regarding the molecular classification subgroup for breast cancer, our meta-analysis showed that ctDNA was only significantly associated with DFS in ER+ breast cancer patients; the difference may be explained by insufficient related studies. ctDNA is most often measured by PCR, ddPCR or NGS. Compared to the latter two, PCR is both widely available and cost-effective. Our results indicate that when detected by PCR, ctDNA was a predictor of poor prognosis in breast cancer patients.

Our meta-analysis of the clinical significance of ctDNA presence at baseline and after therapy suggests that the presence of ctDNA at these time points is an independent poor prognostic factor that is not significantly influenced by systemic therapy. Our present results also indicate that ctDNA detection post-therapy is useful for monitoring therapeutic effects and has prognostic relevance. Similar to ctDNA presence after therapy, ctDNA presence at baseline is predictive of an unfavorable outcome, suggesting that most ctDNA is resistant to current breast cancer therapies. However, we could not evaluate the effects of therapy on the prognostic value of ctDNA, which is an important parameter in prognostic studies [45], due to the variability of therapeutic regimens used in the studies included in our meta-analysis.

Our meta-analysis has confirmed that ctDNA with TP53 mutations in exons 5-8 and ESR1 mutations in amino acids 537 or 538 is associated with poor prognosis among breast cancer patients. Therefore, we can speculate that ctDNA with TP53 mutations in exons 5-8 and ESR1 mutations in amino acids 537 or 538 in breast cancer patients may be an important indicator of worse outcome. Although these results are promising, the clinical relevance of ctDNA with TP53 mutations in exons 5-8 and ESR1 mutations in amino acids 537 or 538 in breast cancer patients remains to be determined, especially since analyses of ctDNA with TP53 mutations in exons 5-8 and ESR1 mutations in amino acids 537 or 538 in patients with other cancers are lacking. In addition, ctDNA may have promising clinical applications in the early detection of breast tumors, as a predictor of minimal residual disease after curative resection, in monitoring disease progression after chemotherapy and in categorizing patients who are at high risk of recurrence so that low-risk patients can avoid unnecessary systemic therapies [24, 46, 47]. In the future, large prospective clinical trials are required to facilitate the implementation of ctDNA detection into daily practice.

Notably, this was the first meta-analysis and systematic review of ctDNA for the prediction of prognosis among breast cancer patients. However, there are several limitations to this study. First, our meta-analysis included only 16 studies. We searched only published data and did not contact the authors of excluded studies for further clarification. Additionally, studies with negative results are often not published, which can cause publication bias [48], potentially causing the correlation between ctDNA and unfavorable clinical outcomes to be overstated. Second, we extracted only summarized population-level data rather than individual patient-level data. Additionally, the HRs of some studies were estimated indirectly as previously reported [49]. These data were less reliable compared to data directly extracted from the original literature. Third, moderate heterogeneity exists among studies; thus, the results should be interpreted cautiously. Moreover, the subgroup analysis indicated that the gene mutation type was a potential source of heterogeneity. Fourth, the sample sizes of several selected articles were small, which may have influenced the diagnostic accuracy of the ctDNA test. Last, the different methods of ctDNA detection may be a potential source of bias.

In summary, our meta-analysis suggests that TP53 mutations in exons 5-8 and ESR1 mutations in amino acids 537 or 538 in ctDNA are potential prognostic biomarkers, biomarkers that may be used to monitor disease progression and promising therapeutic targets for advanced breast cancer.

\section{MATERIALS AND METHODS}

\section{Literature search}

The meta-analysis was performed using the guidelines presented in the Preferred Reporting Items for Systematic Reviews and Meta-Analyses (PRISMA) [50] as shown in Supplementary Table 1. A comprehensive literature search was carried out using the PubMed and Embase databases with the following search criteria: "circulating tumor DNA" or "cell-free DNA" and "mutation" and "breast cancer" for studies published prior to May 1, 2017. Detailed search strategies for the above two databases are presented in Supplementary Table 2. Additionally, manual searches were conducted by reviewing the reference lists of relevant publications.

\section{Selection criteria}

The eligibility of the identified articles was independently reviewed by two authors (Y.G. and L.C.). The criteria for the selection of eligible articles were as follows: (1) articles that reported survival data on breast cancer patients stratified by ctDNA status (presence/ positive and absence/negative) and (2) articles that provided sufficient data for determining estimated hazard ratios (HRs) and 95\% confidence intervals (CIs) for OS and PFS/DFS. 
We selected only articles that were published in peer-reviewed journals and excluded reviews, letters and meeting abstracts. In the case of multiple publications reporting the same or overlapping data, only the most recent study or the study using the largest population were included, as recommended by $\mathrm{Wu}$ et al. [51]. In cases where a study reported insufficient data, the authors were contacted, and if no response was received, the study was excluded.

\section{Quality assessment}

Two authors (Y.G. and L.C.) independently evaluated the quality of the selected literature using the Newcastle-Ottawa Scale (NOS) criteria [52]. A score system was developed based on the NOS criteria, and the total scores ranged from 0 (worst) to 9 (best) for each study (Supplementary Table 3). Disagreement between assessments was resolved by consensus with a third reviewer (X.L.).

\section{Data extraction and outcomes}

First, we extracted the publication information (i.e., name of the first author, year of publication and country) and the characteristics of the participants (i.e., total sample size, tumor stage, ctDNA detection method, mutated genes and sampling time). Next, the HR values with CIs for OS and PFS/DFS were directly extracted from the original enrolled studies or were calculated with the formula suggested by Tierney and colleagues [53]. Additionally, if any study provided only figures without detailed data, we attempted to contact the authors, and if there was no response, we used the Engauge Digitizer 4.1 program (M. Mitchell, Engauge Digitizer, http://markummitchell.github.io/engauge-digitizer/) to extract data from the figures. This program can read exact values by digitizing data points from an image file after manually setting the coordinate axis.

The analyzed endpoints of clinical outcome were OS and PFS/DFS. In patients with early-stage breast cancer (M0), the effect of ctDNA detection on DFS was evaluated, whereas in patients with metastatic breast cancer (M1), the PFS endpoint was evaluated; the effect of ctDNA detection on OS was analyzed for both groups. Additional subgroup analyses were conducted based on the detection method, molecular classification, tumor stage, mutated genes and blood-sampling time point.

\section{Statistical analysis}

RevMan 5.3 software provided by the Cochrane Collaboration was used to conduct all of the statistical analyses, following the introduction of the Cochrane Collaboration for meta-analysis. Heterogeneity among the articles was assessed using the chi-square test and expressed as the " $I^{2}$ " value. In addition, heterogeneity was considered when $P<0.05$ and $/$ or $I^{2}>50 \%$, and the random-effects model was applied; otherwise, the fixedeffects model was used. Subgroup analyses were conducted to explore potential sources of heterogeneity, including the detection method, molecular classification, tumor stage, mutated genes and blood-sampling time point. In addition, a sensitivity analysis, in which one study was omitted at a time from the summary estimate, was conducted to assess whether individual studies significantly influenced the summary statistic. A funnel plot was also constructed by Review Manager Version 5.3 software to evaluate publication bias. Multivariate data analysis was preferred when the study contained data from both univariate and multivariate analyses. HRs and associated $95 \%$ CIs were used to quantify the influence of ctDNA on DFS and OS. A combined HR $>1$ indicated worse survival among breast cancer patients. All statistical tests were two-sided, and $P<$ 0.05 was regarded as statistically significant, except in the heterogeneity analysis.

\section{CONFLICTS OF INTEREST}

There are no conflicts of interest related to this study.

\section{FUNDING}

This work was supported by grants from the National Natural Science Foundation of China (No. 81372813).

\section{REFERENCES}

1. Jemal A, Bray F, Center MM, Ferlay J, Ward E, Forman D. Global cancer statistics. CA Cancer J Clin. 2011; 61:69-90.

2. Openshaw MR, Page K, Fernandez-Garcia D, Guttery D, Shaw JA. The role of ctDNA detection and the potential of the liquid biopsy for breast cancer monitoring. Expert Rev Mol Diagn. 2016; 16:751-5.

3. Shah M, Denlinger CS. Optimal post-treatment surveillance in cancer survivors: is more really better? Oncology (Williston Park).2015; 29:230-40.

4. Lee CH, Dershaw DD, Kopans D, Evans P, Monsees B, Monticciolo D, Brenner RJ, Bassett L, Berg W, Feig S, Hendrick E, Mendelson E, D'Orsi C, et al. Breast cancer screening with imaging: recommendations from the Society of Breast Imaging and the ACR on the use of mammography, breast MRI, breast ultrasound, and other technologies for the detection of clinically occult breast cancer. J Am Coll Radiol. 2010; 7:18-27.

5. Morrow M, Waters J, Morris E. MRI for breast cancer screening, diagnosis, and treatment. Lancet. 2011; 378:1804-11.

6. Crowley E, Di Nicolantonio F, Loupakis F, Bardelli A. Liquid biopsy: monitoring cancer-genetics in the blood. Nat Rev Clin Oncol. 2013;10:472-84. 
7. Bettegowda C, Sausen M, Leary RJ, Kinde I, Wang Y, Agrawal N, Bartlett BR, Wang H, Luber B, Alani RM, Antonarakis ES, Azad NS, Bardelli A, et al. Detection of circulating tumor DNA in early- and late-stage human malignancies. Sci Transl Med. 2014; 6:224ra24.

8. Diaz LA Jr, Williams RT, Wu J, Kinde I, Hecht JR, Berlin J, Allen B, Bozic I, Reiter JG, Nowak MA, Kinzler KW, Oliner KS, Vogelstein B. The molecular evolution of acquired resistance to targeted EGFR blockade in colorectal cancers. Nature. 2012; 486:537-40.

9. Shaw JA, Stebbing J. Circulating free DNA in the management of breast cancer. Ann Transl Med. 2014; 2:3.

10. Diaz LA Jr, Bardelli A. Liquid biopsies: genotyping circulating tumor DNA. J Clin Oncol. 2014; 32:579-86.

11. Jahr S, Hentze H, Englisch S, Hardt D, Fackelmayer FO, Hesch RD, Knippers R. DNA fragments in the blood plasma of cancer patients: quantitations and evidence for their origin from apoptotic and necrotic cells. Cancer Res. 2001; 61:1659-65.

12. Mouliere F, Rosenfeld N. Circulating tumor-derived DNA is shorter than somatic DNA in plasma. Proc Natl Acad Sci USA. 2015; 112:3178-79.

13. De Mattos-Arruda L, Caldas C. Cell-free circulating tumor DNA as a liquid biopsy in breast cancer. Mol Oncol. 2016; 10:464-74.

14. De Mattos-Arruda L, Cortes J, Santarpia L, Vivancos A, Tabernero J, Reis-Filho JS, Seoane J. Circulating tumor cells and cell-free DNA as tools for managing breast cancer. Nat Rev Clin Oncol. 2013; 10:377-89.

15. Beaver JA, Jelovac D, Balukrishna S, Cochran R, Croessmann S, Zabransky DJ, Wong HY, Toro PV, Cidado J, Blair BG, Chu D, Burns T, Higgins MJ, et al. Detection of cancer DNA in plasma of patients with early-stage breast cancer. Clin Cancer Res. 2014; 20:2643-50.

16. Dawson SJ, Tsui DW, Murtaza M, Biggs H, Rueda OM, Chin SF, Dunning MJ, Gale D, Forshew T, MahlerAraujo B, Rajan S, Humphray S, Becq J, et al. Analysis of circulating tumor DNA to monitor metastatic breast cancer. N Engl J Med. 2013; 368:1199-209.

17. Garcia JM, Garcia V, Silva J, Peña C, Dominguez G, Sanchez A, Sanfrutos L, Provencio M, Millan I, Chaparro D, España P, Bonilla F. Extracellular tumor DNA in plasma and overall survival in breast cancer patients. Genes Chromosomes Cancer. 2006; 45:692-701.

18. Liang DH, Ensor JE, Liu ZB, Patel A, Patel TA, Chang JC, Rodriguez AA. Cell-free DNA as a molecular tool for monitoring disease progression and response to therapy in breast cancer patients. Breast Cancer Res Treat. 2016; 155:139-49.

19. Oshiro C, Kagara N, Naoi Y, Shimoda M, Shimomura A, Maruyama N, Shimazu K, Kim SJ, Noguchi S. PIK3CA mutations in serum DNA are predictive of recurrence in primary breast cancer patients. Breast Cancer Res Treat. 2015; 15:299-307.
20. Schiavon G, Hrebien S, Garcia-Murillas I, Cutts RJ, Pearson A, Tarazona N, Fenwick K, Kozarewa I, LopezKnowles E, Ribas R, Nerurkar A, Osin P, Chandarlapaty S, et al. Analysis of ESR1 mutation in circulating tumor DNA demonstrates evolution during therapy for metastatic breast cancer. Sci Transl Med. 2015;7:313ra182.

21. Madic J, Kiialainen A, Bidard FC, Birzele F, Ramey G, Leroy Q, Rio Frio T, Vaucher I, Raynal V, Bernard V, Lermine A, Clausen I, Giroud N, et al. Circulating tumor DNA and circulating tumor cells in metastatic triple negative breast cancer patients. Int J Cancer. 2015; 136:2158-65.

22. Gyanchandani R, Kota KJ, Jonnalagadda AR, Minteer T, Knapick BA, Oesterreich S, Brufsky AM, Lee AV, Puhalla SL. Detection of ESR1 mutations in circulating cell-free DNA from patients with metastatic breast cancer treated with palbociclib and letrozole. Oncotarget. 2017; 8:6690166911. https://doi.org/10.18632/oncotarget.11383.

23. Chandarlapaty S, Chen D, He W, Sung P, Samoila A, You D, Bhatt T, Patel P, Voi M, Gnant M, Hortobagyi G, Baselga J, Moynahan ME. Prevalence of ESR1 Mutations in CellFree DNA and Outcomes in Metastatic Breast Cancer: A Secondary Analysis of the BOLERO-2 Clinical Trial. JAMA Oncol. 2016; 2:1310-1315.

24. Garcia-Murillas I, Schiavon G, Weigelt B, Ng C, Hrebien S, Cutts RJ, Cheang M, Osin P, Nerurkar A, Kozarewa I, Garrido JA, Dowsett M, Reis-Filho JS, et al. Mutation tracking in circulating tumor DNA predicts relapse in early breast cancer. Sci Transl Med. 2015;7:302ra133.

25. Nakauchi C, Kagara N, Shimazu K, Shimomura A, Naoi Y, Shimoda M, Kim SJ, Noguchi S. Detection of TP53/ PIK3CA Mutations in Cell-Free Plasma DNA From Metastatic Breast Cancer Patients Using Next Generation Sequencing. Clin Breast Cancer. 2016; 16:418-423.

26. Olsson E, Winter C, George A, Chen Y, Howlin J, Tang MH, Dahlgren M, Schulz R, Grabau D, van Westen D, Fernö M, Ingvar $\mathrm{C}$, Rose $\mathrm{C}$, et al. Serial monitoring of circulating tumor DNA in patients with primary breast cancer for detection of occult metastatic disease. EMBO Mol Med. 2015; 7:1034-47.

27. Riva F, Bidard FC, Houy A, Saliou A, Madic J, Rampanou A, Hego C, Milder M, Cottu P, Sablin MP, Vincent-Salomon A, Lantz O, Stern MH, et al. Patient-Specific Circulating Tumor DNA Detection during Neoadjuvant Chemotherapy in Triple-Negative Breast Cancer. Clin Chem. 2017; 63:691-699.

28. Shao ZM, Wu J, Shen ZZ, Nguyen M. p53 mutation in plasma DNA and its prognostic value in breast cancer patients. Clin Cancer Res. 2001; 7:2222-7.

29. Silva JM, Silva J, Sanchez A, Garcia JM, Dominguez G, Provencio M, Sanfrutos L, Jareño E, Colas A, España P, Bonilla F. Tumor DNA in plasma at diagnosis of breast cancer patients is a valuable predictor of disease-free survival. Clin Cancer Res. 2002; 8:3761-6. 
30. Spoerke JM, Gendreau S, Walter K, Qiu J, Wilson TR, Savage H, Aimi J, Derynck MK, Chen M, Chan IT, Amler LC, Hampton GM, Johnston S, et al. Heterogeneity and clinical significance of ESR1 mutations in ER-positive metastatic breast cancer patients receiving fulvestrant. Nat Commun. 2016; 7:11579.

31. Takeshita T, Yamamoto Y, Yamamoto-Ibusuki M, Inao T, Sueta A, Fujiwara S, Omoto Y, Iwase H. Prognostic role of PIK3CA mutations of cell-free DNA in early-stage triple negative breast cancer. Cancer Sci. 2015; 106:1582-9.

32. Takeshita $\mathrm{T}$, Yamamoto $\mathrm{Y}$, Yamamoto-Ibusuki M, Inao T, Sueta A, Fujiwara S, Omoto Y, Iwase H. Clinical significance of monitoring ESR1 mutations in circulating cell-free DNA in estrogen receptor positive breast cancer patients. Oncotarget. 2016; 7:32504-18. https://doi. org/10.18632/oncotarget.8839.

33. Cancer Genome Atlas Network. Comprehensive molecular portraits of human breast tumors. Nature. 2012; 49:61-70.

34. Nik-Zainal S, Van Loo P, Wedge DC, Alexandrov LB, Greenman CD, Lau KW, Raine K, Jones D, Marshall J, Ramakrishna M, Shlien A, Cooke SL, Hinton J, et al., and Breast Cancer Working Group of the International Cancer Genome Consortium. The life history of 21 breast cancers. Cell. 2012; 149:994-1007.

35. Shah SP, Morin RD, Khattra J, Prentice L, Pugh T, Burleigh A, Delaney A, Gelmon K, Guliany R, Senz J, Steidl C, Holt RA, Jones S, et al. Mutational evolution in a lobular breast tumour profiled at single nucleotide resolution. Nature. 2009; 46:809-13.

36. Stephens PJ, Tarpey PS, Davies H, Van Loo P, Greenman C, Wedge DC, Nik-Zainal S, Martin S, Varela I, Bignell GR, Yates LR, Papaemmanuil E, Beare D, et al, and Oslo Breast Cancer Consortium (OSBREAC). The landscape of cancer genes and mutational processes in breast cancer. Nature. 2012; 486:400-4.

37. Ciriello G, Gatza ML, Beck AH, Wilkerson MD, Rhie SK, Pastore A, Zhang H, McLellan M, Yau C, Kandoth C, Bowlby R, Shen H, Hayat S, et al, and TCGA Research Network. Comprehensive molecular portraits of invasive lobular breast cancer. Cell. 2015; 163:506-19.

38. Merenbakh-Lamin K, Ben-Baruch N, Yeheskel A, Dvir A, Soussan-Gutman L, Jeselsohn R, Yelensky R, Brown M, Miller VA, Sarid D, Rizel S, Klein B, Rubinek T, et al. D538G mutation in estrogen receptor-alpha: a novel mechanism for acquired endocrine resistance in breast cancer. Cancer Res. 2013; 73:6856-64.

39. Robinson DR, Wu YM, Vats P, Su F, Lonigro RJ, Cao X, Kalyana-Sundaram S, Wang R, Ning Y, Hodges L, Gursky A, Siddiqui J, Tomlins SA, et al. Activating ESR1 mutations in hormone-resistant metastatic breast cancer. Nat Genet. 2013; 45:1446-51.
40. Toy W, Shen Y, Won H, Green B, Sakr RA, Will M, Li Z, Gala K, Fanning S, King TA, Hudis C, Chen D, Taran T, et al. ESR1 ligand-binding domain mutations in hormoneresistant breast cancer. Nat Genet. 2013; 45:1439-45.

41. Olivier M, Hainaut P. TP53 mutation patterns in breast cancers: searching for clues of environmental carcinogenesis. Semin Cancer Biol. 2001; 11:353-60.

42. Borresen-Dale AL. TP53 and breast cancer. Hum Mutat. 2003; 21:292-300.

43. Rossner P Jr, Gammon MD, Zhang YJ, Terry MB, Hibshoosh H, Memeo L, Mansukhani M, Long CM, Garbowski G, Agrawal M, Kalra TS, Gaudet MM, Teitelbaum SL, et al. Mutations in p53, p53 protein overexpression and breast cancer survival. J Cell Mol Med. 2009; 13:3847-57.

44. Clatot F, Augusto L, Di Fiore F. ESR1 mutations in breast cancer. Aging (Albany NY). 2017; 9:3-4. https://doi. org/10.18632/aging.101165.

45. McShane LM, Altman DG, Sauerbrei W, Taube SE, Gion M, Clark GM; Statistics Subcommittee of the NCIEORTC Working Group on Cancer Diagnostics. Reporting recommendations for tumor markers prognostic studies. $\mathrm{J}$ Clin Oncol. 2005; 23:9067-72.

46. Haber DA, Velculescu VE. Blood-based analyses of cancer: circulating tumor cells and circulating tumor DNA. Cancer Discov. 2014; 4:650-61.

47. De Mattos-Arruda L, Caldas C. Cell-free circulating tumour DNA as a liquid biopsy in breast cancer. Mol Oncol. 2016; 10:464-74.

48. Crawford JM, Briggs CL, Engeland CG. Publication bias and its implications for evidence-based clinical decision making. J Dent Educ. 2010; 74:593-600.

49. Greenland S. Quantitative methods in the review of epidemiologic literature. Epidemiol Rev. 1987; 9:1-30.

50. Moher D, Liberati A, Tetzlaff J, Altman DG, and PRISMA Group. Preferred reporting items for systematic reviews and meta-analyses: the PRISMA statement. Int J Surg. 2010; 8:336-341.

51. Wu N, Xu B, Xiang Y, Wu L, Zhang Y, Ma X, Tong S, Shu M, Song Z, Li Y, Zhong L. Association of inflammatory factors with occurrence and recurrence of atrial fibrillation: A meta-analysis. Int J Cardiol. 2013; 169:62-72.

52. Wells GA, Shea B, O'Connell D, Peterson J, Welch V, Losos M, Tugwell P. The Newcastle-Ottawa Scale (NOS) for assessing the quality of nonrandomized studies in metaanalyses. 2011. Available: http:/www.ohri.ca/programs/ clinical_epidemi ology/oxford.asp.

53. Tierney JF, Stewart LA, Ghersi D, Burdett S, Sydes MR. Practical methods for incorporating summary time-to-event data into meta-analysis. Trials. 2007; 8:16. 